\title{
On the Use of Design Patterns in Collaborative Design Processes
}

\author{
Claudia Iacob, Ernesto Damiani \\ Department of Computer Technology \\ University of Milan \\ Via Comelico, 39-41 \\ Milan, Italy \\ \{claudia.iacob, ernesto.damiani\}@unimi.it
}

\begin{abstract}
Even if the usefulness of a knowledge repository represented as a collection of design patterns is largely recognized in the literature, little work has been done in investigating and measuring the impact such a collection would have on collaborative design processes involving designers. The paper describes the results of a case study designed to bring some insight into the matter. 18 design workshops were conducted with 18 teams of undergraduate students in Computer Science. Making use of a collection of design patterns for the design of synchronous applications, they were asked to design the GUI and the interaction process of applications which support synchronous collaboration in activities such as drawing, text editing, game solving, and searching. To answer the questions addressed by the case study, the results of the workshops were triangulated from: i) audio recordings of the conversations of each team, ii) notes taken on the participants interactions by a facilitator present during the workshops, and iii) feedback provided by each participant through a questionnaire, at the end of each workshop.
\end{abstract}

\section{Keywords}

Design patterns, collaborative design.

\section{INTRODUCTION}

The origin of the concept of design pattern dates back to the "70s when the architect Christopher Alexander defined it as follows: "Each pattern describes a problem which occurs over and over again in our environment, and then describes the core of the solution to that problem, in such a way that you can use this solution a million times over, without ever doing it the same way twice" [2]. Alexander proposed a collection of interrelated design patterns for architectural design and envisioned them being used: 1) as knowledge repositories containing the solutions of often recurring urban design problems, 2) as means of

Permission to make digital or hard copies of all or part of this work for personal or classroom use is granted without fee provided that copies are not made or distributed for profit or commercial advantage and that copies bear this notice and the full citation on the first page. To copy otherwise, or republish, to post on servers or to redistribute to lists, requires prior specific permission and/or a fee.

DESIRE'11, October 19-21, 2011, Eindhoven, the Netherlands

Copyright (C) 2010 ACM ISBN 978-1-4503-0754-3... \$10.00. communication of solutions among the communities of architects and, 3) as communication means between architects and their clients in the design of urban spaces.

Soon after, the concept has been adopted in other fields such as software engineering [14] and Human-Computer Interaction (HCI) [3, 24]. Also, patterns have been extensively used in teaching $[4,18]$, bridging communication gaps between users and designers [8], and abstracting results of ethnographic studies of cooperative work $[19,21]$.

Several HCI collections of patterns are now available. Mostly, they address web user interface design [24], interactive exhibits [3], user interface related programming, hypermedia applications, or ubiquitous computing [6,20]. Moreover, design patterns have been proposed for the design of social interfaces [7], groupware technology [22], web accessibility [13], and cross-culture collaboration [21].

The adoption of the concept of design patterns in other fields led to new and innovative ways of using them. On the one hand, software engineering applies design patterns for expressing Object-Oriented software design experience [14]. On the other hand, HCI designers adopted the design pattern approach to document and describe "the reasons for design decisions and the experience from past projects, to create a corporate memory of design knowledge" [3].

Today, the usefulness of a knowledge repository represented as a collection of design patterns is largely recognized $[9,11,15]$, but little work has been done in investigating and measuring the impact such a collection would have on collaborative design processes involving designers. This paper aims at providing additional insight into the matter by describing a case study designed to answer the following questions: a) Are the format and the content of an existing collection of design patterns easy to understand for novice software designers?, b) Given a collection of design patterns targeting a design area, how do novice designers make use of it during collaborative design processes?, c) What pattern representation would best fit for working with a collection of design patterns?, d) What is the overall impact of using design patterns in collaborative design processes?, e) What strategies do novice designers develop in working with a collection of design patterns? 
The paper is structured as follows: first, it provides an overview of the work related to the topic of the paper; then, it briefly describes the collection of design patterns which was used during the case study as subject of evaluation; further on, the paper describes the case study in terms of its objectives, the methodology used, and its results. Lastly, the limitations of this work are presented.

\section{DOCUMENTED USES AND EVALUATIONS OF DESIGN PATTERNS \\ Teaching}

Findings of teaching and evaluating Computer Science courses that dealt with HCI design patterns are summarized in [4]. They suggest that HCI design patterns are useful tools to teach HCI design principles as well as to support students in formulating their own design experiences. Two major ways of using design patterns for teaching have been identified. Firstly, taught as a method, they should be considered "as a segment of a larger advanced class in HCI design methodologies". Secondly, design patterns serve well as a tool and format for teaching HCI design principles.

A specific added value for the use of design patterns is identified in [18] as being the support in acquiring design skills and domain knowledge. The three case studies described in [18] support the following propositions: a) "Novices will faster gain understanding in problem solving and design skills, when they learn to design with the design patterns approach first, before they learn to understand entire systems", b) "Experienced designers will not experience a learning effect from the use of design patterns, but might find them useful in other ways", and c) "Training novices with the use of design patterns will increase the quality of the schemas they build to represent a system".

\section{Design}

An initial and emerging collection of 45 pre-patterns for ubiquitous computing have been described and evaluated in [6]. Sixteen (16) pairs of designers used the pre-patterns for designing location-enhanced applications. The pre-patterns were emailed to the participants prior to a 90-minute design sessions. The design sessions were directly observed, results showing evidence of the following: a) pre-patterns helped novice designers, b) pre-patterns helped designers with the unfamiliar domain, and c) pre-patterns helped designers avoid some design problems.

An extension to this work is presented in [20] where the same collection of pre-patterns was evaluated by 22 pairs of professional designers. Half of the pairs performed a 120-minute design creation task without any external aid, while the other half was given access to the pre-pattern collection via a browser for performing the same task. Results show that the pre-patterns were mostly effective in supporting designers generate design ideas, and allowing them to go back to the pre-patterns to get clarifications on open issues.

A slightly different approach to evaluating design patterns is described in [8] where the contribution that a collection of interrelated patterns could make to the user participation in the design of interactive systems is investigated. A designer-facilitator worked with a user to develop the design of either a travel website or a web-based learning resource using a collection of design patterns addressing web design. Direct observations revealed that users made extensive reference to the patterns' illustrations, often without referring to the text of the patterns. Also, it proved to be important that only a small number of patterns were presented to the users at the same time. The patterns were also used as a checklist to ensure that all the issues have been discussed.

If design patterns emphasize capturing a problem-solution pair in a certain context, design claims focus on describing the positive and the negative implications of a design decision [1]. The case study described in [1] evaluates the benefits of structuring design advice in a pattern or a claim form and, instead of declaring the pattern or the claim structure as a clear winner, proposes a hybrid structure for sharing design advice. The paper also underlines an underappreciated contribution of design patterns which is their ability to offer "a way to capture and share successful design trade-offs in context" [1].

\section{A COLLECTION OF PATTERNS FOR THE DESIGN OF SYNCHRONOUS APPLICATIONS}

In order to assess the impact of the use of design patterns in collaborative design processes, an already defined collection of design patterns has been used. This collection addresses the design of software applications which support synchronous collaboration. The section describes the method used for mining for the patterns together with a brief description of the patterns identified.

\section{Mining for the Patterns}

The mining method used for identifying our design patterns consisted in two phases: 1) the analysis of the results of the design processes followed by 13 teams of professional designers (1 team), graduate students in Computer Science (9 teams), and undergraduate students in Computer Science (3 teams) throughout a series of design workshops, and 2) the analysis of 20 applications existing on the market (developed as either research projects or commercial products) which support synchronous collaboration in activities such as drawing, text editing, searching, and games. The two phases were developed independently of one another, their results being triangulated after both phases were conducted [16].

During a design workshop, a team of 3-5 designers was asked to design the GUI and the interaction process for an application in the domain of the mining process and the design issues they address were collected. To support the results of the workshops, an additional set of software applications in the area of the mining process were analyzed in order to identify in what measure the design issues discussed during the workshops were considered in the implementation of existing applications.

The most recurring design issues in both the workshops' results and the results from the analysis of the software 
applications were considered to be documented as design patterns [17].

\section{The Pattern Collection}

The mining process led to the identification of 15 design patterns. Each design pattern was further on described by: a). a name which expresses the main point addressed by the pattern, b). a unique $I D$ which is a numerical value used to uniquely identify each pattern, c). a set of keywords associated to the pattern, d). a descriptive illustration transmitting the main idea of the pattern, e). the description of the context in which the pattern can be applied, f). the problem addressed by the pattern, g). the set of forces which influence this problem and express the tradeoffs to be considered when applying the pattern, h). the description of a solution to tackle the problem, i). a set of symptoms which might require the application of the pattern, $\mathrm{j}$ ). a set of consequences that the application of the pattern triggers, $\mathrm{k}$ ). examples of the application of the pattern in existing software applications, 1). possibly other related patterns.

The brief description of all the patterns identified is presented below.

Who is the coordinator? addresses the problem of providing a coordination mechanism which: a). allows all collaborators to take part in the collaboration and b). maintains the resource in a consistent state at all times.

Integrated chat addresses the problem of supporting the communication among collaborators, suggesting the integration of an instant messaging feature in the design of the application.

Eyes wide open addresses the problem of allowing each collaborator to be notified about and visualize what the others are contributing to the process at any time.

Choose your collaborators suggests allowing each user to be able to choose the people s/he wants to work with during the collaboration.

Collaboration, always social suggests integrating mechanisms of tagging, ranking, annotating, and commenting in the application in order to support the collaborators in forming a community.

With or without collaboration addresses the issue of providing users with an additional private area, not available to the other collaborators, where each collaborator has total control and where $\mathrm{s} / \mathrm{he}$ is provided with tools specific to the context of the application.

My contribution addresses the problem of supporting the identification of each individual's contribution to the collaborative process.

Track history of collaboration suggests saving the history of the collaborative process and making it available through repositories, $\log$ files, or timelines.

Adapt application to device suggests supporting the materialization of the application on various devices so that users are allowed to collaborate even if they use different devices for that.
Annotate suggests allowing users to enhance the shared resource with textual, audio, or video notes on the misunderstandings, additional explanations, or inquiries they might have. Any annotation is in itself a thread-like entity, allowing the collaborators to answer back through text, audio, or video material.

Support versioning indicates enhancing the application with a versioning mechanism able to support the collaborators in viewing and editing older versions of the document they are working on.

Collaborative undo suggests supporting the users in undoing changes performed on the shared document, maintaining the resource consistent.

Customize collaboration points to providing the collaborators with the possibility of customizing the parameters of their collaborative process. These parameters could be simple visualization or editing options, or more complex options such as assigning roles and rights among the collaborators.

Shared summary suggests providing the collaborators with an automatic way to create summaries of their collaborative processes. These summaries could include intermediary results of their process, statistical data, or simplified versions of the shared resource.

Resume collaboration suggests allowing the collaborators to pause their collaborative process, store its state, and restore it later without affecting any collaborator's contribution.

\section{CASE STUDY}

This section presents a case study conducted for identifying the impact a collection of design patterns addressing the design of synchronous applications has on the collaborative design of such applications by novice software designers. 18 design workshops were conducted with 18 teams of undergraduate students in Computer Science. Making use of the patterns described above, they were asked to design the GUI and the interaction process of an application to support synchronous collaboration in activities such as drawing, text editing, game solving, and searching. Their processes were audio recorded, a facilitator observed their interactions, and each participant provided his/her feedback on the workshop through a questionnaire. The section introduces the reader to the overall objectives of the case study, the methodology used, the results obtained, and a discussion on the implications these results have on collaborative design processes.

\section{Objectives}

The objective of the case study is to answer the following research questions:

a) Are the format and the content of our existing collection of design patterns easy to understand for novice software designers?

b) Having available a collection of design patterns targeting a design area, how do novice designers make use of it during collaborative design processes? 
c) What pattern representation would best fit for working with a collection of design patterns?

d) What is the overall impact of using design patterns in collaborative design processes?

e) What strategies do novice designers develop in working with a collection of design patterns?

Moreover, the outcomes of the case study (i.e. transcripts, sketches, facilitator's notes) represent valuable data to be mined for in an attempt to extend the collection of patterns.

\section{Method}

\section{Participants}

The total number of participants was 75 , out of which $75 \%$ were male, and $25 \%$ were female. 66 of them $(88 \%)$ were first year students in Computer Science, 8 (11\%) were following their second year, while 1 of them was a third year student in Design. Out of the 75 participants, only two have had prior experience with working with design patterns. Solely 9 of the participants $(12 \%)$ had more than 3 years experience with designing software applications, while the rest of $88 \%$ were novice software designers, with less than 3 years experience in software design.

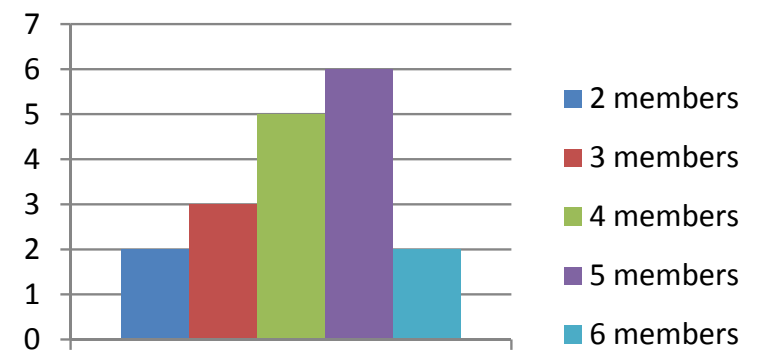

No. of teams

Figure 1 - Groups member distribution

The 75 participants were divided into 18 teams with the member distribution of each team depicted in Figure 1. The majority of the teams were formed of 5 participants, while 2 of the teams were formed of 2 participants each.

\section{Procedure}

Participants worked in teams of 2-6 and the duration of a workshop was 2 hours. There was one facilitator present during each of the workshops, her role being to: a). introduce the participants to the workshop, b). walk them through each phase of the workshop, c). take notes of their interactions, and d). observe them throughout the workshop and support them if needed.

Each team was presented with a brief overview of the goals of the workshop and with the collection of the 15 patterns described above. Each pattern was represented on a paper card, being described by its name, its unique $I D$, the set of keywords associated to it, a representative illustration, the problem addressed by the pattern, and the solution proposed to tackle the problem (Figure 2). The restrictive description was mainly enforced by the paper card representation of the patterns and by the time length of each workshop.

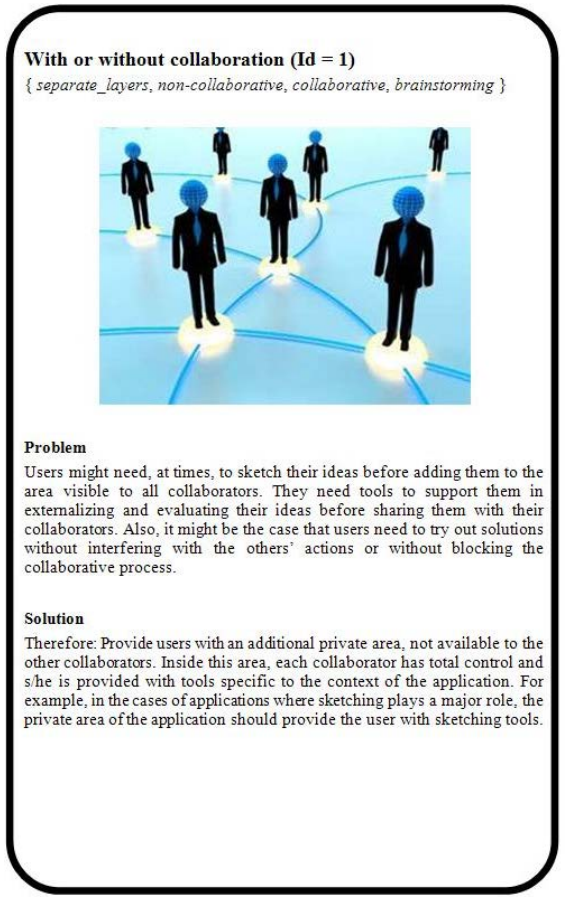

Figure 2 - Paper card representation of the patterns

The initial phase of each workshop asked the participants to go through the patterns and to get familiar with them. No strategy was suggested, all of the teams being free to follow their own approach for looking the patterns over. All the misunderstandings or unclear issues were discussed with the facilitator and collected for further analysis.

As a second phase, each team was presented with a list of problems and was encouraged to choose one problem for which to design, using the patterns provided, a software application. The problems addressed different areas of synchronous collaboration, such as collaborative drawing, collaborative text editing, collaborative game solving, or collaborative search.

The designs were meant to consider solely the GUI and the interaction process of the application. To support their design processes, the teams were encouraged to answer the following questions: a) who are the users targeted?, b) what is the motivation of the users' collaboration?, c) when and where can the application be used?, and d) how can the users interact with the application? [5]. Also, they were asked to sketch their ideas, express all the design problems they encounter and, possibly, create a mock up of their overall design. Their conversations were recorded and a facilitator observed all of the teams, taking notes of their interactions.

Lastly, each participant answered a questionnaire providing feedback on the overall process followed and on the use of the patterns.

\section{Problems}

The list of problems proposed during the workshops included collaborative drawing, collaborative text editing, collaborative search, and collaborative game solving. 
The problem of collaborative drawing asked for the design of a software application which would allow painters, graphic designers and/or visual artists to collaboratively create one diagrammatic representation. The problem of collaborative text editing required participants to design an application which would allow a group of users to create a summary of a written text in a synchronous collaborative fashion.

The set of games considered for collaborative solving consisted of puzzles and crosswords. The common requirement for both was that more users solve one game in the same time. The problem of collaborative search required that more users are able to perform one websearch from remote locations.

\section{Measures}

The objective of the case study is to answer a set of open questions. For each of the questions, measures were defined and used to quantify the final results.

a) Are the format and the content of our existing collection of design patterns easy to understand for novice software designers?

Participants were asked to choose the most useful and the least useful pattern defining element (i.e. name, ID, keywords, picture, problem, solution) in understanding the patterns. Moreover, they were asked to order the pattern defining elements from the most useful element to the least useful element in supporting the understandability of the presented patterns.

After having gone through all the patterns and having used them, the participants were asked to rate on a Likert-type scale (with 1 - not at all understandable, 2 - not understandable, 3 - I can't say, 4 - understandable, and 5 very understandable) the degree of understandability of each of the patterns presented. The patterns were available to the participants throughout the rating. The average rate was computed for each of the patterns, and a global rate was calculated for identifying the overall understandability of the whole collection.

b) Having available a collection of design patterns targeting a design area, how do novice designers make use of it during collaborative design processes?

Through the questionnaire, the participants were asked to rate on a Likert-type scale (with 1 being not at all useful, 2 - not useful, 3 - I can't say, 4 - useful, and 5 - very useful) the degree of usefulness of the patterns for each of the following documented uses of patterns [6, 11, 20]: 1) understanding the design space of the application, 2) searching for design problems, 3) searching for solutions for already identified design problems, 4) communicating with the other members of the team, 5) remembering similar design situations encountered, and 6) brainstorming for design ideas for the application. For each of the above activities, the average rate of the answers was computed.

Moreover, the feedback from the participants tried to identify which of the above activities was mostly supported by each of the patterns. c) What pattern representation would best fit for working with a collection of design patterns?

After having used the patterns in their paper card representation, the participants were asked to choose which, in their opinion, would be the most suitable representation for a collection of patterns having as options the following: i) paper cards, ii) wiki applications, iii) search engines, and iv) specialized application with personalized features.

d) What is the overall impact of using design patterns in collaborative design processes?

The overall impact of using the patterns was measured by: 1) the Likert-type scale (with 1 being not at all useful, 2 not useful, 3 - I can't say, 4 - useful, and 5 - very useful) ratings participants assigned for each of the patterns with respect to the usefulness of the pattern in the overall process, and

2) the qualitative feedback provided by the participants as answer to the open ended question: "How have the patterns supported your design process?".

e) What strategies do novice designers develop in working with a collection of design patterns?

The conversations of all the teams were recorded and transcribed. Their dialogues were divided into sentences (i.e. small fragments of dialogues - usually lines of the dialogues - related to a particular concept or action), all those sentences containing references to the patterns provided being filtered and considered for further analysis. The coding scheme used for coding the sentences referencing patterns classified these sentences as indicating: 1) browsing the collection, 2) reading a pattern, 3) using a solution, 4) adapting a pattern, 5) modifying a pattern, 6) searching for a pattern, 7) explaining a pattern to another member of the team, 8). re-referencing a pattern, and 9) generating a design idea inspired by a pattern.

The strategies used by the teams were abstracted from: a) the sequences of actions (i.e. those defined by the coding scheme) they followed in isolated contexts of their design processes, b) the ratio of the sentences in each category over the total number of sentences considered, and c) the facilitator's notes on the participants' interactions.

\section{Results and Discussion}

The results of this case study are described in terms of: a) the understandability, usage, and modifiability of the patterns, b) the participants' feedback, c) the identified strategies participants developed while working with the patterns, and d) the possible implications these findings have on using design patterns in collaborative design processes.

\section{Understanding, Using, and Modifying the Patterns Understandability}

The problem and the solution described by each pattern were the elements considered the most useful in understanding a design pattern, $51 \%$ of the participants rating them as such. 


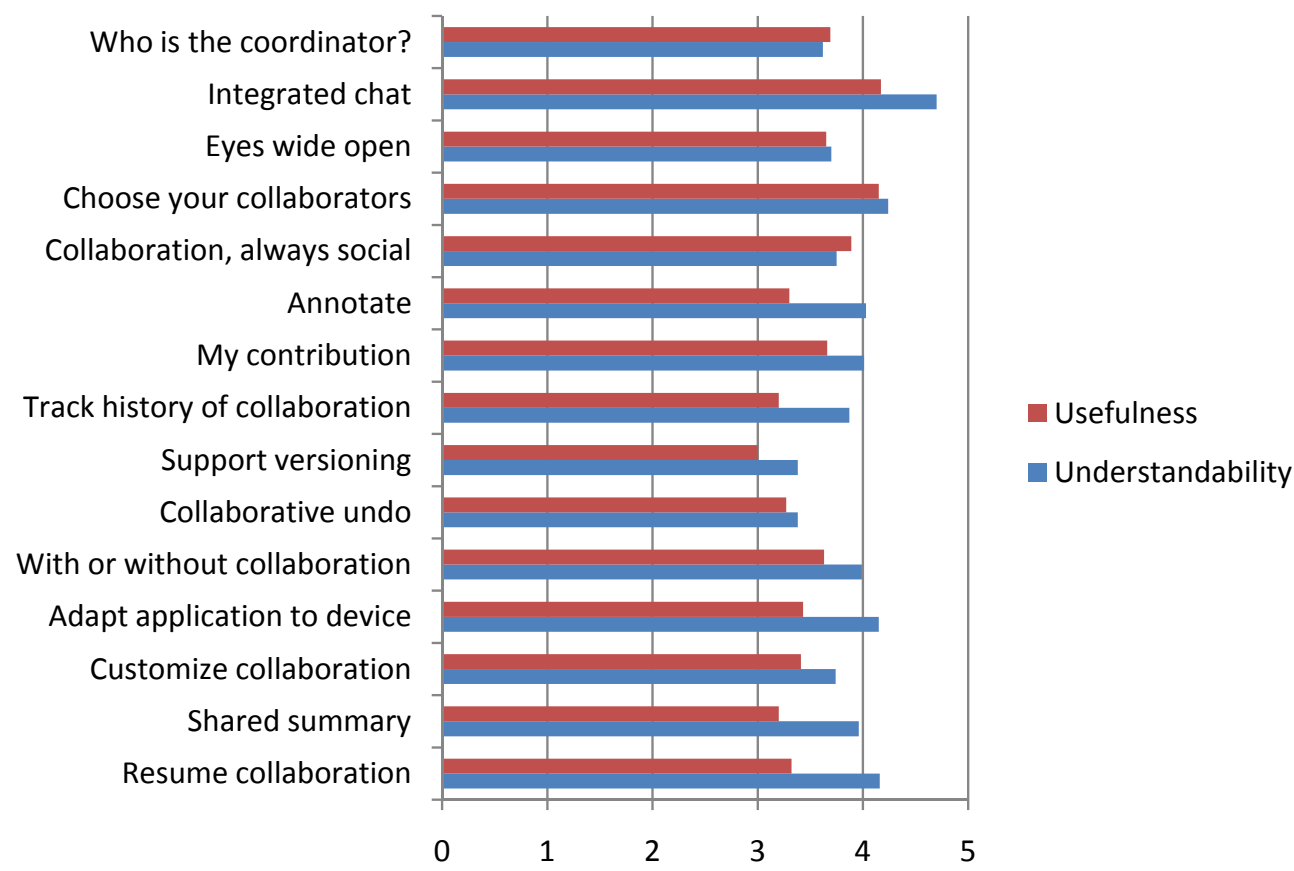

Figure 3 - Understandability and usefulness rates for each of the patterns

On the other hand, $78.7 \%$ of the participants found the unique ID of each pattern as the least useful element for understanding patterns. As expected, a relatively large number of participants $(32.4 \%)$ found the name of the patterns helpful. However, even if the illustration assigned to each pattern was expected to help the participants grasp the main idea of each pattern, results showed little evidence of the usefulness of this element.

The average rate of understandability of the collection of patterns provided was 3.91 (S.D. 0.341) on a scale from 1 to 5 (with 5 being very understandable), proving that, overall, the participants faced little trouble in grasping the idea of each pattern and its usefulness. The complete information on the rate of understandability of each pattern is depicted in Figure 3.

\section{Usage}

As answer to the question "To what extent were the patterns useful for the following (on a Likert-type scale, with 1 - not at all useful, 2 - not useful, 3 - I can't say, 4 useful, and 5 - very useful)?", participants rated:

a) Searching for documented problems with an average rate of 4.28 (with $46.7 \%$ of the participants rating it as very useful),

b) Searching for solutions for already identified problems with an average rate of 4.13 (with $45.9 \%$ of the participants rating it as very useful),

c) Communicating with other members in your team, 3.93 (with $44 \%$ of the participants rating it as very useful), d) Brainstorming for design ideas for the application under design, 3.79 (with $34.6 \%$ of the participants rating it as useful),

e) Understanding the design space of the application, 3.48 (with $25.3 \%$ of the participants rating it as very useful),

f) Remembering similar design situations previously encountered, 3.32 (with $26.7 \%$ of the participants rating it as very useful).

Moreover, some patterns proved specifically useful for some of the above mentioned actions, according to the data provided in Figure 4. For example, much debate has been around the pattern "Choose your collaborators", the participants trying to come up with solutions for allowing users to start their collaborative process and to choose the users they want to work with. The pattern "My contribution" reminded the participants of several contexts which request the identification of one individual's contribution and of existing applications which support this action. Also, throughout their design processes, the participants mostly searched for solutions for the problems of: a) adapting the application to a specific device, b) allowing the undo operation on a collaboratively edited resource, and c) versioning.

\section{Modifiability}

Sixty-one (61) of the participants $(81.33 \%)$ mentioned that the information provided for defining each pattern was enough and that no additional information would be needed. Eight $(8)$ of the participants $(10.66 \%)$ would have found a set of examples of application of each pattern useful in better understanding the idea of each pattern. 


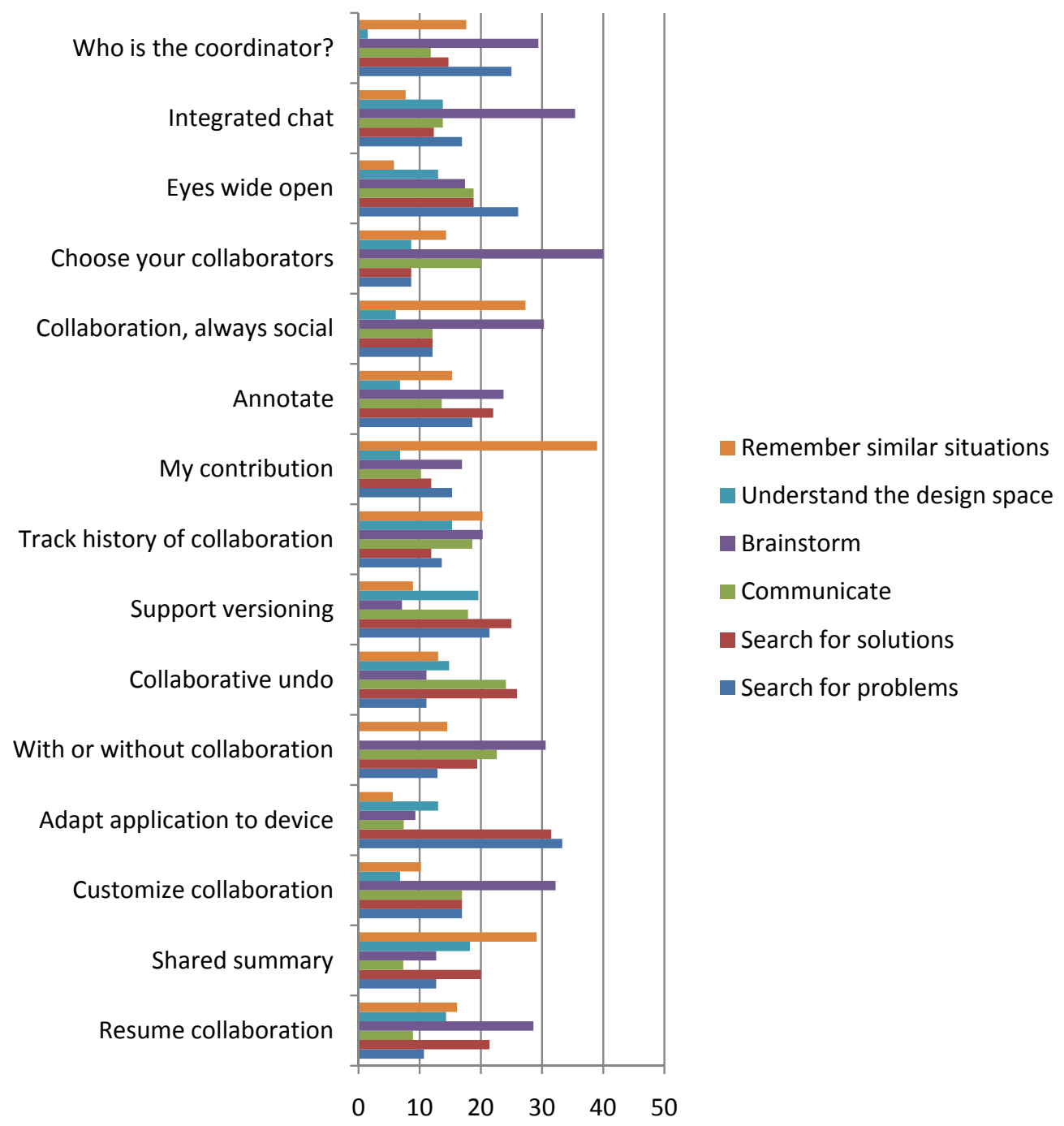

Figure 4 - Degree of usage (in percentage) of each pattern for specific actions

Two (2) of the participants $(2.66 \%)$ suggested adding some more details in the description of each pattern, while keeping the same defining elements. Lastly, one of the participants $(1.33 \%)$ suggested adding a defining element for each pattern able to list down some frequently asked questions related to the pattern.

Having to choose a representation for the collection of patterns which would best suit collaborative design processes, $62.5 \%$ of the participants opted for the paper card representation, $28.8 \%$ chose a search engine application, $13.9 \%$ opted for a wiki-like application, while only $4.2 \%$ of the participants considered the option of a specialized application to work with design patterns.

Overall Feedback

Asked to rate the overall usefulness of the patterns, participants provided an average rate of the collection (computed as the mean of the usefulness rates of all the patterns - Figure 3 ) of 3.53 out of 5 (S.D. 0.346).
As support for the above mentioned quantitative data, some of the answers to the open question "How have the patterns supported your design process?" included:

i) "They [the patterns] helped us in searching possible problems. We analyzed all of them to check how each pattern applies to our design process."

ii) "They [the patterns] were very useful in the beginning of the workshop for understanding what should we consider and in which way. Also, during the work, they helped us maintain a coherent and detailed line of work."

iii) "They were fundamental in guiding us through the realization of the project. They helped us consider things that we wouldn't have considered without them."

iv) "The patterns allowed the discussion among the group members and the exchange of ideas." 
v) "The patterns provided indications and a precise schema on which to reason for solving the problems encountered during the workshop."

\section{Strategies Developed}

Abstracting from a) the sequences of actions the teams performed on the collection of patterns in isolated contexts of their design processes, b) the ratio of each category of actions the teams performed, and c) the facilitator's notes on the participants' interactions, a set of strategies the participants developed while using the design patterns were identified.

\section{Customize Pattern Identification}

In going through the patterns and trying to get familiar with the problems addressed by them, the teams often tried to associate each pattern with a characteristic word. Having done that, their dialogs would contain references to the patterns through the words associated to them (e.g. "We can decide on a fixed time for all the game and during the game one can take maximum 2 breaks, and then we look into the solution for the pause one [the pattern Resume collaboration]"). Interesting enough, these words were not consciously chosen from the list of keywords provided in the description of the patterns. However, with the exception of one case, all the words the teams associated with the patterns already belonged to the list of keywords provided by the cards.

Two of the teams filtered the collection of patterns after going through it and discussing it once and chose a subset of these patterns they considered fundamental for their design process. Throughout their work, they referred mostly to these patterns.

\section{Signal Patterns}

Often times, while some of the members of a team were focusing on the design task, the other(s) browsed the collection of patterns and tried to relate the team's design decisions to the solutions proposed by the patterns. When the team member(s) browsing the patterns identified a useful pattern at a specific moment, s/he signaled this pattern to the team.

Some examples of such references are: "Ok, there is a thing I read here [My contribution]: for understanding who has placed a certain piece", or "Look at this, this is interesting [points to pattern With or without collaboration] When you solve a puzzle you should have a private area where you try out the pieces and when a piece works well where it is placed, you just add it to the whole puzzle".

\section{Search - Analyze - Apply}

The most common strategy the teams were expected to choose consisted in: a) initiate by writing down possible problems they would face, b) browse the collection of patterns searching for those patterns documenting the problems they considered, c) point to a pattern once found and read it, d) analyze the solutions proposed by the pattern and assess which solution to apply.
Contrary to the expectations, less than half of the teams adopted this precise path of actions. However, all of the teams performed at least two of these actions during their design processes.

\section{The Pattern Collection as a Checklist}

Eight out of the 18 teams used the collection of patterns also as a checklist. They initiated their work after going through the patterns, but initially ignored them. After reaching an idea for the application they were designing and sketching a draft of it, they went through all the patterns, one by one, in order to make sure that they covered all the issues addressed by the collection. For each of the patterns, they analyzed whether they considered the issue addressed by the pattern or not. In the affirmative case, they identified the solution they adopted. Such an example is: "We used this one with the updates [pattern Eyes wide open], and we sent notifications". In the negative case, they explained the reasons for which the pattern did not apply to their design context. An example of such a reference is: "The pause one [pattern With or without collaboration], we skip it?/ Yes, we have included that in the chat feature".

\section{Patterns as Startup Tools}

Four of the teams initiated their design processes by going through the patterns, one by one, and identifying how each pattern could be applied in the context of their application's design. Then, when faced with a problem during their design process, the teams tried to remember which of the patterns addressed that problem. Examples of such references are: "Yes, there was a pattern on that", or "There was one [pattern] that was mentioning the saving... because if we are 5 and we decide to save, we should be able to do that". Moreover, specific situations faced during the design process reminded the teams of the patterns they browsed at the beginning of the process. As example of such a reference, consider "Exactly, this was one of the issues in the patterns. If one clicks on the piece and drags it, in that moment that piece is locked'.

\section{Patterns as Source of Inspiration}

A common behavior of all the teams was to consult the patterns ever so often during their design processes. This helped them explore their design options and take informed decisions on the solutions to consider applying. Moreover, once going through the patterns, the teams considered problems and design ideas they wouldn't have considered otherwise. Patterns inspired the teams in adding elements to their designs, and some example of references to such situations are: "Let's add something about notifications [after reading Eyes wide open]", or "How do they choose the collaborators? [pointing to the pattern Choose your collaborators]".

\section{Mark the Use}

The final result provided by each team was a sketch or a mockup of their overall design. No strategy was suggested to the participants for marking the patterns used. However, there were three ways they decided to address this. The 
majority of the teams grouped together all the patterns they used, putting them aside. Others have decided to arrange the patterns in the order they used them throughout the process. A more systematic approach was adopted by two of the teams which annotated their sketches with the IDs of the patterns they used, marking the use of each pattern in a specific context of the application's design.

\section{What do you mean?}

Patterns were often used as means of making oneself understood. The teams used the patterns in order to explain each other concepts or to discuss open issues or misunderstandings. For example, one of the most challenging concepts to grasp was reverting changes, the teams making use of the Collaborative undo pattern to explain each other the concept and the way it can be addressed in the context of the applications they were designing. Similar results have been identified in [11].

\section{Beyond Patterns}

During their work with the patterns, some of the teams went beyond the definition provided by the cards and pointed out examples of applications of the patterns in software systems commonly used. Moreover, one of the teams identified possible relationships existing between patterns. For example, they considered the patterns Track history of collaboration, Collaborative undo, and Support versioning related to each other, even if they did not specify exactly in which way these patterns are related. A similar association was identified among the patterns Collaboration, always social, Annotate, and Customize collaboration.

\section{Implications}

The strategies described above trigger a set of implications to the use of design patterns in collaborative design processes.

- Initiating by going through a problem-solution knowledge repository related to the design domain allows the designers to frame their ideas, better understand the further implications of their early design decisions, and be inspired in their work.

- As searching in such a repository is the most common action designers are expected to perform, the representation of such a knowledge base should consider including a straightforward way of querying it. In addition to that, browsing the collection proves to be frequent mostly at the beginning of the design process. This asks for efficient and user-friendly visualization features and tools.

- Using patterns collaboratively, designers should be able to signal patterns to one another, supporting them in sharing knowledge.

- $\quad$ Marking the use of the patterns directly on the design result (sketch, mockup) allows documenting design processes, supporting their review and understandability.

- A collection of often recurring design problems and their solutions (i.e. a design pattern collection) may be used as a checklist to support validating design results and decisions.

\section{THREATS TO VALIDITY}

Even if they have been sporadically criticized for "offering a poor basis for generalization" [10], case studies are powerful empirical methods used mainly for exploratory investigations. Using them at their full potential implies defining their objectives, the criteria for interpreting the findings, and their limitations [12, 23]. The latter consist in exploring and identifying the validity of the design and the results of the case study.

One of the limitations of the case study presented by the paper refers to not involving a sufficiently large number of professional designers. The small percentage of experienced designers $(12 \%$ of the participants had more than 3 years experience in software design) is not convincing enough for any generalization of the results to professional designers. Therefore, all the implications the results trigger address mainly novice software designers.

The patterns provided to the designers addressed a particular domain - the design of synchronous collaborative applications. Moreover, the collection contained a relatively small number of patterns -15 . However, as support for the findings brought to light by the case study, the results presented in [11] identify several similar points even if the collection of patterns used by the authors addressed web design and contained 22 design patterns. In [11], Diaz et al. identified the "Read one-by one" browsing strategy and defined it as "participants went through all the patterns as a first strategy to identify candidates and look for ideas". Also, the web patterns proved to be intuitive and easily understood by the designers involved.

Strong conclusions with respect to generalizations of the use of design patterns in collaborative design processes ask for further empirical work. Nevertheless, this work aims at bringing more knowledge to the matter and provides a starting point for further understanding and investigation.

\section{CONCLUSIONS AND FUTURE WORK}

The paper describes a case study conducted in order to identify the impact of using a collection of design patterns in collaborative design processes. 18 teams of novice designers were asked to get familiar with and use a collection of 15 design patterns for the design of synchronous applications. They were given a design task to design the GUI and the interaction process of an application which supports synchronous collaboration in one of the areas: drawing, searching, text editing, games and were allowed to work on it for 2 hours.

The conversations of all the teams were recorded, each participant provided his/her feedback at the end of the process through a questionnaire, and a facilitator present during all the workshops took notes of the participants interactions. Based on that, the following were derived:

- The major actions the participants performed using the patterns were: a) searching for documented problems (average rate of 4.28/5), b) searching for solutions for already identified problems (average rate of 
4.13/5), and c) communicating with other members in your team (average rate of 3.93/5).

- $\quad 62.5 \%$ of the participants opted for the paper card representation of the patterns, while $28.8 \%$ chose a search engine application for the same purpose.

- $\quad$ The patterns proved to be both easy to understand in the format presented and useful during the design process.

In addition to that, 9 strategies developed by the participants while using the patterns were abstracted from the data collected.

As future work, the authors are interested in involving professional software designers in such collaborative processes and comparatively analyze their strategies and their feedback with those obtained from the current study.

\section{ACKNOWLEDGMENTS}

Special thanks to all the participants in the workshops for the inspiring ideas and great designs.

\section{REFERENCES}

1. Abraham, G., Atwood, M. E. 2009. Patterns or claims: do they help in communicating design advice?. Proceedings of OZCHI '09. ACM, New York, NY, USA, 25-32.

2. Alexander, C., Ishikawa, S., Silverstein, M. 1977. A pattern language: Towns, buildings, construction. New York: Oxford University Press

3. Borchers, J. 2001. A Pattern Approach to Interaction Design. John Wiley \& Sons, Inc.

4. Borchers, J. 2002. Teaching HCI Design Patterns: Experience From Two University Courses. Patterns in Practice: AWorkshop for UI Designers, CHI2002, Minneapolis, MI, Apr, 2002.

5. Carroll, J.M. 1995. Scenario-Based Design: Envisioning Work and Technology in System Development. John Wiley \& Sons, Inc., New York, NY, USA, 1995

6. Chung, E.S., Hong, J. I., Lin, J., Prabaker, M. K., Landay, J. A., Liu, A. L. 2004. Development and evaluation of emerging design patterns for ubiquitous computing. Proceedings of DIS '04. ACM, New York, NY, USA, 233-242.

7. Crumlish, C., Malone, E. 2009. Designing Social Interfaces. O'Reilly Media, Inc.

8. Dearden, A., Finlay, J., Allgar, E., Mcmanus, B. 2002. Using Pattern Languages in Participatory Design. Proceedings of the Participatory Design Conference (PDC 2002). CPSR, Palo Alto, CA., 2002.

9. Dearden, A., Finlay, J. 2006. Pattern languages in HCI: A critical review. Human Computer Interaction Journal, 21 (1) March, pp.49-102.

10. Dewayne E. Perry, Susan Elliott Sim, and Steve M. Easterbrook. 2004. Case Studies for Software
Engineers. Proceedings of the 26th International Conference on Software Engineering (ICSE '04). IEEE Computer Society, Washington, DC, USA, 736-738.

11.Diaz, P., Rosson, M.B., Aedo, I., Carroll, J.M. 2009. Web Design Patterns: Investigating User Goals and Browsing Strategies. Proceedings of the International Symposium on End-User Development (IS-EUD '09), Springer-Verlag, Berlin, Heidelberg, 186-204.

12. Flyvbjerg, B. 2006. Five Misunderstandings about Case-Study Research. Qualitative Inquiry, 12(2), April, 2006, pp. 219-245.

13. Fogli, D. Parasiliti Provenza, L., Bernareggi, C. 2010. A design pattern language for accessible web sites, Proceedings of AVI 2010, pp. 307-310.

14. Gamma, E., R. Helm, R. Johnson, Vlissides, J. 1995. Design Patterns: Elements of Reusable Object-Oriented Software. Reading, MA: Addison-Wesley.

15.Herrmann, T., Hoffmann, M., Jahnke, I., Kienle, A., Kunau, G., Loser, K. U., Menold, N. 2003. Concepts for usable patterns of groupware applications. Proceedings of the ACM SIGGROUP conference on Supporting group work (GROUP '03). ACM, New York, NY, USA, 349-358.

16. Iacob, C. 2011. A Design Pattern Mining Method for Interaction Design, Proc. EICS2011, 217-222.

17. Iacob, C. 2011. Designing Systems for Synchronous Collaboration: From Collaborative Software to Design Patterns, EuroPLoP 2011.

18. Kolfschoten, G., Lukosch, S., Verbraeck, A., Valentin, E., de Vreedea, G.J. 2010. Cognitive learning efficiency through the use of design patterns in teaching. Computers\&Education, 54(3), April 2010, pp. 652-660.

19. Martin, D., Rouncefield, M., Sommerville, I. 2002. Applying patterns of cooperative interaction to work (re)design: e-government and planning. Proceedings of CHI '02. ACM, New York, NY, USA, 235-242.

20. Saponas, T.S., Prabaker, M. K, Abowd, G.D., Landay, J. A. 2006. The impact of pre-patterns on the design of digital home applications. Proceedings of DIS '06. ACM, New York, NY, USA, 189-198.

21. Schadewitz, N. 2009. Design Patterns for Cross-cultural Collaboration. International Journal of Design, 3(3).

22. Schummer, T. and S. Lukosch. 2007. Patterns for Computer-Mediated Interaction. John Wiley \& Sons, Ltd.

23. Tellis, W. 1997. Introduction to Case Study, The Qualitative Report, 3, 2, 1997, pp. 1-11.

24. Tidwell, J. 2005. Designing Interfaces: Patterns for Effective Interaction Design. O'Reilly Media. 